\title{
POSSIBLE GENERATION OF A $\pi$-CONDENSATION IN FREE SPACE BY COLLISIONS BETWEEN PHOTONS AND PROTONS
}

\author{
QI-REN ZHANG \\ School of Physics, Peking University, Beijing 100871, China
}

\begin{abstract}
A sharply peaked structure is found in the angular distribution of emitted $\pi^{+}$mesons from the photon-proton collisions. It offers a possible way for generating a $\pi^{+}$-condensation in free space. To make the stimulated emission of $\pi^{+}$-mesons efficient, a ring resonator is designed.

Key words: Hadron quantum electrodynamics,Stimulated $\pi^{+}$-emission, $\pi$-condensation in a free space. Ring resonator.
\end{abstract}

PACS number(s): 13.60.Le, 03.70.+k, 03.75.Nt, 29.20.db

\section{INTRODUCTION}

Possible $\pi$-condensation in nuclear matter was first considered in seventies last century [1, 2]. To put the work on a better mathematic foundation we exactly solved the Dirac equation for the nucleon in a classical $\pi$-field[3] . A theory of nuclear matter was therefore proposed in the form of relativistic mean field theory, in which a mean $\pi$ field is taken into account [4-7]. To minimize the energy per-nucleon, a nonzero value of mean $\pi$-field appears. It shows the possible existence of the $\pi$-condensation in nuclear matter. $\pi$-condensation is also considered in quarkgluon plasma 8 -10]. However, until now we have not seen any direct experimental evidence for the existence of a $\pi$ condensation, neither in nuclear matter nor in free space. It makes us eager to generate a $\pi$-condensation experimentally, in free space first. Of course, the generation of a $\pi$-condensation in free space itself is interesting.

We designed a way for generating a $\gamma$-ray laser using the photon-electron collisions [11, 12]. It is because we see that, the angular distribution of the outgoing $\gamma$-photons is sharply peaked. Since the energy of an emitted $\gamma$ photon is a definite function of its outgoing direction, the $\gamma$-photons are emitted almost into one state. In this paper, we shall show that a similar situation appears for the $\pi^{+}$-meson emissions in the photon-proton collisions. The angular distribution of the emitted $\pi^{+}$-mesons is also sharply peaked. We may therefore design a similar way for generating a $\pi^{+}$-condensation in free space, by use of photon-proton collisions.

\section{ANGULAR DISTRIBUTION OF THE EMITTED $\pi^{+}$-MESONS IN A HEAD ON PHOTON-PROTON COLLISION}

Consider the reaction

$$
\gamma+\mathrm{p} \rightarrow \pi^{+}+\mathrm{n}
$$

in which a $\gamma$-photon and a proton p head on collide with each other, and transit into a $\pi^{+}$-meson and a neutron $n$. When the energy of the photon is not too high, the colour degrees of freedom in hadrons are not important. The problem may therefore be handled by hadron quantum electrodynamics. Denote the proton field, neutron field, charged meson field and photon field by $\Psi_{p}, \Psi_{n}, \Phi$ and $\left(A_{\mu}\right)$ respectively, the Lagrangian density for the system is

$$
\mathcal{L}=\mathcal{L}_{p}+\mathcal{L}_{n}+\mathcal{L}_{m}+\mathcal{L}_{\gamma}+\mathcal{L}_{s}
$$

in which

$$
\begin{aligned}
\mathcal{L}_{p} & =-\bar{\Psi}_{p}\left[\gamma_{\mu}\left(\partial_{\mu}-\mathrm{i} e A_{\mu}\right)+m\right] \Psi_{p} \\
\mathcal{L}_{n} & =-\bar{\Psi}_{n}\left(\gamma_{\mu} \partial_{\mu}+m\right) \Psi_{n} \\
\mathcal{L}_{m} & =-\left[\left(\partial_{\mu}+\mathrm{i} e A_{\mu}\right) \Phi^{*}\left(\partial_{\mu}-\mathrm{i} e A_{\mu}\right) \Phi+m_{\pi}^{2} \Phi^{*} \Phi\right] \\
\mathcal{L}_{\gamma} & =-\frac{1}{4}\left(\partial_{\mu} A_{\nu}-\partial_{\nu} A_{\mu}\right)\left(\partial_{\mu} A_{\nu}-\partial_{\nu} A_{\mu}\right)
\end{aligned}
$$

and

$$
\mathcal{L}_{s}=\mathrm{i} \sqrt{2} G\left(\bar{\Psi}_{p} \gamma_{5} \Psi_{n} \Phi+\bar{\Psi}_{n} \gamma_{5} \Psi_{p} \Phi^{*}\right)
$$

are Lagrangian densities of protons with their electromagnetic interactions, neutrons, $\pi^{ \pm}$-mesons with their electromagnetic interactions, photons, and the strong interactions between related hadrons, respectively. The nature unit system of $\hbar=c=1$ is used. Symbols are defined in the usual way as given in standard text books, for examples in [13, 14]. $e$ and $G$ are interaction constants for electromagnetic interaction and strong interaction respectively, with the corresponding values $\alpha \equiv e^{2} / 4 \pi=1 / 137 . \cdots$ and $\alpha_{s} \equiv G^{2} / 4 \pi=14.6$.

For the reaction (1), a factor of electromagnetic interaction appears always with a factor of strong interaction. The constants $\alpha$ and $\alpha_{s}$ always appear together in the form of a product $\alpha \alpha_{s}$. Since $\alpha \alpha_{s}<1$, a perturbation treatment for the reaction seems reasonable. The lowest order transition matrix element for the reaction is

$$
\begin{aligned}
& \left\langle\sigma_{n}, \boldsymbol{q}, \boldsymbol{\kappa}|T| \boldsymbol{k}, \boldsymbol{e}, \boldsymbol{p}, \sigma_{p}\right\rangle=-G e \frac{\prod_{\mu=0}^{3} \delta\left(p_{\mu}+k_{\mu}-q_{\mu}-\kappa_{\mu}\right)}{\sqrt{2}(2 \pi)^{2} \sqrt{k \kappa_{0}}} \\
& \times \bar{u}_{\sigma_{n}}(\boldsymbol{q}) \gamma_{5}\left[\frac{1}{\mathrm{i} \gamma^{\mu}\left(p_{\mu}+k_{\mu}\right)+m} \mathrm{i} \gamma^{\mu} e_{\mu}\right. \\
& \left.+\frac{1}{\left(p^{\mu}-q^{\mu}\right)\left(p_{\mu}-q_{\mu}\right)+m_{\pi}^{2}} 2 \kappa^{\mu} e_{\mu}\right] u_{\sigma_{p}}(\boldsymbol{p})
\end{aligned}
$$

in which $\left[p_{\mu}\right],\left[q_{\mu}\right],\left[k_{\mu}\right]$, and $\left[\kappa_{\mu}\right]$, with $\mu=0,1,2$, and 3 , are energy-momentum four vectors of proton, neutron, 
photon, and $\pi^{+}$-meson respectively. $\left[e_{\mu}\right]$ with $\mu=$ $0,1,2$, and 3 , is the polarization four vector of the photon. $m$ and $m_{\pi}$ are masses of the nucleon and the charged pion respectively. $u_{\sigma_{n}}(\boldsymbol{q})$ is the Dirac spinor of the neutron with spin $\sigma_{n}$ and momentum $\boldsymbol{q}$, and $u_{\sigma_{p}}(\boldsymbol{p})$ is that of the proton with spin $\sigma_{p}$ and momentum $\boldsymbol{p}$. $\delta$-functions in (8) show energy-momentum conservation in the reaction (11). They, together with the energy-momentum relations $p_{0}=\sqrt{p^{2}+m^{2}}, q_{0}=\sqrt{q^{2}+m^{2}}, \kappa_{0}=\sqrt{\kappa^{2}+m_{\pi}^{2}}$ and $k_{0}=k$, give

$$
\begin{aligned}
& \left(A^{2}-1\right) \kappa^{2}+2 A B \kappa+B^{2}-m_{\pi}^{2}=0 \\
& A=\frac{k-p}{p_{0}+k} \cos \theta \text { and } B=\frac{m_{\pi}^{2}+2 k\left(p_{0}+p\right)}{2\left(p_{0}+k\right)},
\end{aligned}
$$

$\theta$ is the angle between moving directions of the incident photon and the emitted pion. $\kappa>0$ is the absolute value of the pion momentum, therefore should be the positive root of equation (9). It defines the energy $\kappa_{0}$ as a function of the moving direction $\theta$ for the pion.

Take the Coulomb gauge, in which the contribution from longitudinal and temporal components of the electromagnetic field is collected in the coulomb energy between charged particles, and is negligible when space charge effect being unimportant. Only the contribution from the transverse components of the electromagnetic field will be considered in the following. Let $\boldsymbol{e}_{i} \mathrm{e}^{\mathrm{i} \boldsymbol{k} \cdot \boldsymbol{x}}$ with $i=1,2$ show the transverse plane wave, we have $e_{i 0}=0, \boldsymbol{e}_{i} \cdot \boldsymbol{k}=0$ for $i=1,2$, and $\boldsymbol{e}_{i}^{*} \cdot \boldsymbol{e}_{i^{\prime}}=\delta_{i i^{\prime}}$.

For experiments without measuring spins, all transition probabilities and cross-sections have to be summed up over the final spin states and averaged over the initial spin states. Using the projection operator method, we obtain

$$
\begin{aligned}
& \frac{1}{4} \sum_{i=1,2} \sum_{\sigma_{n}=-1,1} \sum_{\sigma_{p}=-1,1}\left|\bar{u}_{\sigma_{n}}(\boldsymbol{q}) \gamma_{5}\left[\frac{1}{\mathrm{i} \gamma^{\mu}\left(p_{\mu}+k_{\mu}\right)+m} \mathrm{i} \gamma^{\mu} e_{i \mu}+\frac{1}{\left(p^{\mu}-q^{\mu}\right)\left(p_{\mu}-q_{\mu}\right)+m_{\pi}^{2}} 2 \kappa^{\mu} e_{i \mu}\right] u_{\sigma_{p}}(\boldsymbol{p})\right|^{2}=\frac{X}{p_{0} q_{0}}, \\
& X=\frac{1}{2} f^{2}\left\{\left[2 k\left(p+p_{0}\right)-\kappa_{0}\left(k+p_{0}\right)+\kappa(k-p) \cos \theta\right]\left[k\left(2 p+p_{0}\right)-p^{2}\right]+2 p k\left(p_{0}+p\right)(p-k+\kappa \cos \theta)\right. \\
&\left.+\left[p^{2}+p_{0}\left(k-\kappa_{0}\right)\right](p-k)^{2}-\left(p^{2}+p_{0} k\right)(p-k)(p-k+\kappa \cos \theta)\right\}+\kappa^{2} g(\theta) \sin ^{2} \theta\left\{f k\left(p+p_{0}\right)\right. \\
&\left.+g(\theta)\left[p_{0}\left(k-\kappa_{0}\right)+p(k-\kappa \cos \theta)\right]\right\},
\end{aligned}
$$

with $f=1 /\left[2 k\left(p_{0}+p\right)\right]$, and $g(\theta)=1 /\left[2 k\left(\kappa \cos \theta-\kappa_{0}\right)\right]$. The transition probability per-unit time for the pion goes into a differential solid angle $\mathrm{d} \Omega$ is

$$
\frac{\mathrm{d} P}{\mathrm{~d} t}=2 \alpha_{s} \alpha \frac{X J \kappa^{2}}{k \kappa_{0} q_{0} p_{0}} \mathrm{~d} \Omega \mathrm{d} \kappa \delta\left(E_{f}-E_{i}\right) .
$$

$J=1 / V$ is the incident photon current density in our unit system, and $V$ is the volume of the reaction space. $E_{i}=p_{0}+k$ and $E_{f}=q_{0}+\kappa_{0}$ are initial and final energies of the process respectively. Under fixed initial momenta $\boldsymbol{p}$ and $\boldsymbol{k}$,

$$
\mathrm{d} \kappa=\frac{\kappa_{0}\left(p_{0}+k-\kappa_{0}\right)}{\left(p_{0}+k\right) \kappa+\kappa_{0}(p-k) \cos \theta} \mathrm{d}\left(E_{f}-E_{i}\right) .
$$

We therefore have

$$
\frac{\mathrm{d} P}{\mathrm{~d} t}=2 \alpha_{s} \alpha X Y J \lambda_{\pi}^{2} \mathrm{~d} \Omega
$$

$\lambda_{\pi}$ is the Compton wavelength of the charged pion, and

$$
Y=\frac{\kappa^{2} m_{\pi}^{2}}{k p_{0}\left[\left(p_{0}+k\right) \kappa+\kappa_{0}(p-k) \cos \theta\right]} .
$$

The differential cross-section for the photo-production of the charged pion on a proton is therefore

$$
\frac{\mathrm{d} \sigma}{\mathrm{d} \Omega}=2 \alpha \alpha_{s} X Y \lambda_{\pi}^{2} .
$$

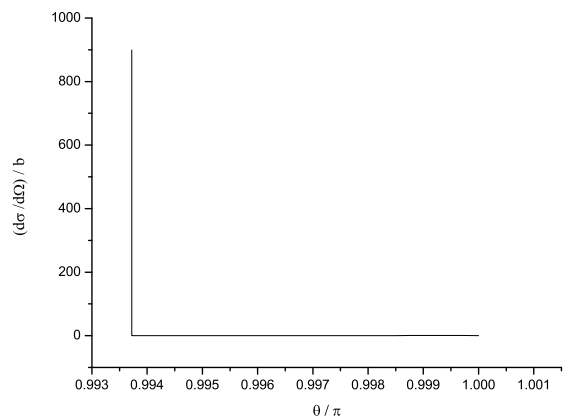

FIG. 1: Relation between the differential cross-section in unit of barn for pion emission and the angle $\theta$ in unit of $\pi$, in a head on collision of an $1.4 \mathrm{MeV}$ photon and a $434 \mathrm{GeV}$ proton

Notice, $X$ and $Y$ are dimensionless.

An example of numerical results is shown in figures 1 and 2, The angular distribution shown in figure 1 is rather characteristic. It is sharply peaked, and is therefore favorable for emitting pions into the most probable state. However, the most probable state is not unique. In the example shown in figure 1, the most probable emis- 


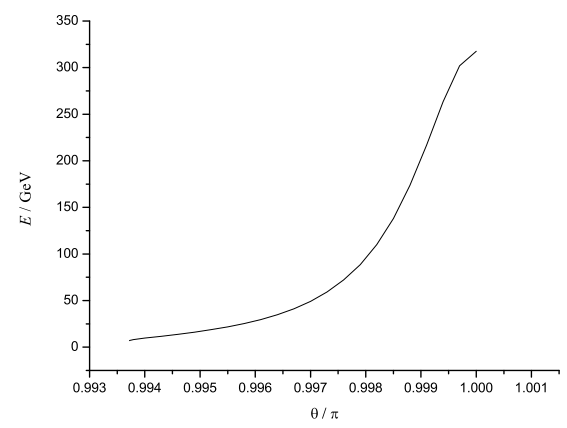

FIG. 2: Relation between the emitted pion energy $E$ in unit of $\mathrm{GeV}$ and the angle $\theta$ in unit of $\pi$, in a head on collision of an $1.4 \mathrm{MeV}$ photon and a $434 \mathrm{GeV}$ proton

sion directions distribute on the surface of a cone, each along a generatrix. The vertex of the cone is at the reaction point, and the axis is on the incident line of the proton. The angle between the generatrix and the axis is $0.006278 \pi$. In the following we shall show that a resonance mechanism makes almost all emitted pions go to one selected most probable state, therefore generates a $\pi^{+}$-condensation in free space.

\section{STIMULATED EMISSION, RESONANCE, AND THE RING RESONATOR FOR THE $\pi$-CONDENSATION}

The key ingredient for making a laser is the stimulated emission of radiation. This is also true for making a $\pi$ condensation in the free space. If there are already $N$ pions in a state, the transition probability for emitting one more pion into this state has to be multiplied by an extra factor $N+1$. The equation (15) is therefore generalized to

$$
\frac{\mathrm{d} P}{\mathrm{~d} t}=2(N+1) \alpha_{s} \alpha X Y J \lambda_{\pi}^{2} \mathrm{~d} \Omega,
$$

which includes contributions of both spontaneous and stimulated emissions of pions into a given state. Here we see a positive feedback between pion numbers of already in and emitted into a given state. The result is a collapse of pion population into the most probable states.

To make emitted pions condense in one state, we need a resonance mechanism. Figure 3 shows a schematic designation of a ring resonator for the stimulated emission of $\pi^{+}$-mesons in head on collisions between photons and protons. The straight line denotes the incident line, and the circle denotes the storage ring. They intersect each other at two gaps on the ring. Photons and protons inject from left and right respectively along the incident line, and are designed to collide with each other at the gaps. Elementary geometry tells us that angles between tangents of the circle at two gaps and the incident line

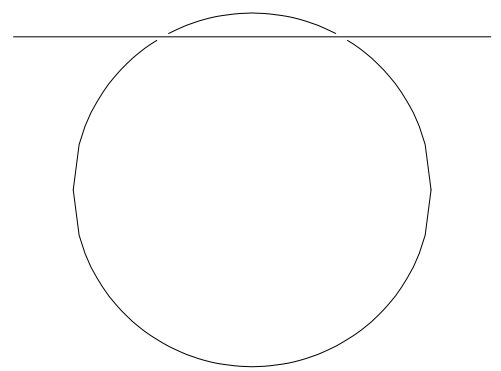

FIG. 3: A schematic diagram of the ring resonator for the stimulated emission of $\pi^{+}$-mesons in head on collisions between photons and protons.

equal each other, so that we may design these tangents along the most probable directions for pion emission at both gaps. $\pi^{+}$-mesons emitted on this most probable direction therefore enter the inner space of the ring. It is its central circular channel. They move along the channel under the interaction of an appropriate constant magnetic field perpendicular to the ring plane. A coincidence of colliding photons and protons together with the earlier emitted $\pi^{+}$-mesons at the gaps is designed, so that the stimulated emissions may happen. The already stored $\pi^{+}$-mesons stimulate the new $\pi^{+}$-meson emissions at the gap. The $\pi^{+}$-mesons are emitted along the most probable direction selected by the ring, and enter the storage ring at the gap under the interaction of the magnetic field. They are therefore prepared to stimulate the next $\pi^{+}$-meson emissions at the next gap. In this way a resonance is formed and a special most probable emission is selected at each gap.

In our example of head on collision between a $1.4 \mathrm{MeV}$ photon and a $434 \mathrm{GeV}$ proton, the energy of the most probable emitted $\pi^{+}$-meson is $6.9 \mathrm{GeV}$, as shown in figure 2. In a magnetic field of $B=1 \mathrm{~T}$, they would move in a storage ring of radius $23 \mathrm{~m}$. Using the data shown at the end of last section, we see that two gaps on the ring in figure 3 open a central angle of $0.012556 \pi$ at the center of the ring. Therefore the length of the arc between these two gaps on the ring of radius $23 \mathrm{~m}$ is $0.9 \mathrm{~m}$. One may therefore open many pairs of gaps on the ring to intensify the condensed pion beam many times in one circle. On the other hand, the half-life time of a $6.9 \mathrm{GeV}$ $\pi^{+}$-meson is $8.95 \times 10^{-7} \mathrm{~s}$. A half of $\pi^{+}$-mesons in the beam may move $268.33 \mathrm{~m}$ before their decay. It is about twice of the ring circumference. It seems, we may generate a rather intense condensed pion beam in this way, and store it in the ring. However, there are various interactions between $\pi^{+}$-mesons. Among them, the long range electromagnetic interaction may be important at not too high density of the meson beam. This is the so called space charge problem. The electric force of the space 
charge is outward perpendicular to the meson beam, and the magnetic force of the moving space charge is inward perpendicular to the meson beam. They together may make nonzero probability for mesons to leave from the resonance orbits, and limit the beam density. It offers a saturation mechanism for the $\pi$-condensation in our example. Fortunately, effects of these electric and magnetic forces kill each other, especially for high velocity mesons. Their total effect approaches zero at the relativity limit $v \rightarrow c$, and therefore makes no serious problem against generating a $\pi^{+}$-condensation with an enough intensity. Finally, charged pions running in a ring may lose energy by Bremsstrahlung. But it may be easily compensated by usual acceleration techniques.

\section{CONCLUSIONS}

The $\pi$-condensation may be generated in a way like that in the laser generation. First of all, we need a pion source. In our example proposed here, it is played by the hadronic reaction (11). Various sharply peaked spectra of pion emission appear. They make emissions concentrate to some specified pion states, and therefore are welcome. We then need a way for realizing the stimulated pion emissions to start the $\pi$-condensation, and need a resonance mechanism for selecting a special pion state to condense. These are designed in the ring resonator shown in the last section.

The reaction (11) was analysed by the hadron electrodynamics. Hadron-dynamics is not a fundamental theory, but an effective theory only. Therefore we should not rely on its quantitative results. We may obtain the quantitative results directly by experiments. However, some qualitative characters do not depend on the dynamical details. In the derivation and the numerical calculation we see, that the sharply peaked structures of the angular distribution for pion emissions are connected directly with the relativistic energy-momentum conservation relations (9) and (10). It is a result of kinetics governing the reaction, and therefore is reliable. The designation of the ring resonator is based on fundamental electromagnetism, and is therefore reliable too. It makes us believe that our proposal is worthy to try experimentally.
[1] Sawyer R. F. 1972 Phys. Rev. Lett. 29382.

[2] Scalapino D. J. 1972 Phys. Rev. Lett. 29386.

[3] Zhang Q.-R. 1981 Phys. Lett. B104 347.

[4] Zhang Q.-R. 1981 Phys. Ener. Fort. Phys. Nucl. (in Chinese) 515.

[5] Zhang Q.-R. 1981 Phys. Ener. Fort. Phys. Nucl. (in Chinese) 5314 .

[6] Zhang Q.-R. and Greiner W. 1995 Mod. Phys. Lett. A10 2809.

[7] Gao C.-Y., Guo H. and Zhang Q.-R., 1999 Int. J. Mod. Phys. E8 39

[8] Son D. T. and Stephanov M. A., 2001 Phys. Rev. Lett.
86592

[9] He L, Jin M. and Zhuang P., 2005 Phys. Rev. D, 71 116001

[10] Cao G., He L. and Huang X. , 2017 Chin. Phys. C41 051001.

[11] Zhang Q.-R. 2014 Chin. Phys. B23 010306.

[12] Zhang Q.-R. 2015 Chin. Phys. B24 054208.

[13] Lurie D. 1968 Particles and Fields, (Interscience Publisher, New York)

[14] Weinberg S. 1995 The Quantum Theory of Fields I, (Cambridge Press, Cambridge UK) 